\title{
3D Study Of Magnetic Fields In NGC 6946
}

\author{
Anna Williams ${ }^{1}$, George Heald ${ }^{2,3}$, Eric Wilcots ${ }^{1}$ and Ellen Zweibel ${ }^{1}$ \\ ${ }^{1}$ Department of Astronomy, University of Wisconsin-Madison, \\ 475 N. Charter Street, Madison, WI, USA \\ email: williams@astro.wisc.edu, ewilcots@astro.wisc.edu, zweibel@astro.wisc.edu \\ ${ }^{2}$ Astron Postbus 2, 7990 AA Dwingeloo, The Netherlands \\ ${ }^{3}$ Kapteyn Astronomical Institute, Postbus 800, 9700 AV, Groningen, The Netherlands \\ email: heald@astron.nl
}

\begin{abstract}
Recent advancements in both radio observatories and computing have opened a new regime of $3 \mathrm{D}$ observations. Not only do these instruments measure emission lines and radio continuum over much larger bandpasses, but they also simultaneously observe the polarized emission over the same large bandpasses with increased sensitivity. This "polarization spectrum" can be used to recover information about the 3D structure of magnetic fields in the universe. Our combined 3-20 cm observations of NGC 6946 taken with the Westerbork Synthesis Radio Telescope provide highly sensitive diagnostics of the internal depolarization across the galaxy. We use model fitting to determine likely mechanisms for depolarization in different regions of the galaxy, and glean information about the coherent and turbulent magnetic fields in NGC 6946. We produce Faraday dispersion maps that illustrate how we can probe different depths into the galaxy at different wavelengths and display new features of the line of sight magnetic field. This work is just a sample of the new 3D studies that are possible with upgraded and new radio instruments like the VLA, ATCA, and SKA.
\end{abstract}

Keywords. galaxies: ISM, magnetic fields, structure

Magnetic fields are important ingredients in the interstellar medium of galaxies. They accelerate cosmic rays, affect star formation, and regulate the redistribution of matter and energy. Despite their ubiquitous presence, their growth and coevolution with galactic processes is not well understood. We use $3-20 \mathrm{~cm}$ polarization observations from the Westerbork Synthesis Radio Telescope to map the turbulent and coherent line-of-sight magnetic fields across the disk of the nearly face-on galaxy NGC 6946. We fit the observed data with the Burn slab model as described by Sokoloff et al. (1998). This simple model considers a slab of magnetoionized medium threaded with a coherent, line-of-sight magnetic field and cells of random magnetic fields. Our results show strong internal Faraday dispersion (Burn slab) in the south-west quadrant where there is little polarized emission at wavelengths $>13 \mathrm{~cm}$, reinforcing the need to consider the $3 \mathrm{D}$ geometry of the magnetic fields in the interstellar medium. We also find strong correspondence between Faraday dispersion and HII regions. For additional information on the model fitting procedure, results, and future work, please go to www.astro.wisc.edu/ williams for the poster PDF.

\section{Acknowledgements}

We acknowledge support from R. Beck (MPIfR), Astron, the National Science Foundation, and NASA.

\section{References}

Sokoloff, D. D., et al. 1998, MNRAS, 299, 189 